\title{
Eigenvalue based SNR Estimation for Cognitive Radio in Presence of Channel Correlation
}

\author{
Shree Krishna Sharma, Symeon Chatzinotas, and Björn Ottersten \\ SnT - securityandtrust.lu, University of Luxembourg \\ Email: $\{$ shree.sharma, symeon.chatzinotas, bjorn.ottersten\}@uni.lu
}

\begin{abstract}
In addition to spectrum sensing capability required by a Cognitive Radio (CR), Signal to Noise Ratio (SNR) estimation of the primary signals is crucial in order to adapt its coverage area dynamically using underlay techniques. Furthermore, in practical scenarios, the fading channel may be correlated due to various causes such as insufficient scattering in the propagation path and antenna mutual coupling. In this context, we consider the SNR estimation problem for a CR in the presence of channel correlation. We study an eigenvaluebased SNR estimation technique for large-scale CR networks using asymptotic Random Matrix Theory (RMT). We carry out detailed theoretical analysis of the signal plus noise hypothesis to derive the asymptotic eigenvalue probability distribution function (a.e.p.d.f.) of the received signal's covariance matrix in the presence of the correlated channel. Then an SNR estimation technique based on the derived a.e.p.d.f. is proposed for PU SNR in the presence of channel correlation and its performance is evaluated in terms of normalized Mean Square Error (MSE). It is shown that the PU SNR can be accurately estimated in the presence of channel correlation using the proposed technique even in low SNR region.

Index Terms: SNR Estimation, Channel Correlation, Cognitive
\end{abstract} Radio, Underlay

\section{INTRODUCTION}

During the last decade, the demand for high speed wireless connections has constantly been increasing due to the proliferation of multimedia services. However, the available frequency resources are becoming scarce due to spectrum segmentation and dedicated frequency allocation of standardized wireless systems. In this context, exploring efficient spectrum sharing techniques to enhance spectral efficiency while guaranteeing quality of service is a highly relevant and challenging problem. Cognitive Radio (CR) is considered a promising candidate for enhancing the spectrum efficiency of wireless communication systems because it is aware of its operating environments and can adjust its parameters dynamically [1]. The most common cognitive techniques in the literature can be categorized into Spectrum Sensing (SS) or interweave, underlay, overlay and database techniques [2]. In SS only techniques, Secondary Users (SUs) are allowed to transmit whenever Primary Users (PUs) do not use that specific band, whereas in underlay techniques, SUs are allowed to transmit as long as they respect the interference constraint of the PUs. Several SS techniques have been proposed in the literature for CR based systems [3-5] and they have different operational requirements, advantages and disadvantages. Among all existing techniques, eigenvaluebased methods are found to be preferable in the presence of noise covariance uncertainty [6,7]. Most of aforementioned methods involve decision statistics calculated based on the eigenvalue distribution of the received signal's covariance matrix and use recent results from Random Matrix Theory (RMT) [8,9].

RMT has been used in the literature in various applications such as modeling transmit/receive correlation in Multiple Input Multiple Output (MIMO) channels and multiuser MIMO fading $[10,11]$. In the existing literature, RMT has received attention in the CR research community, specifically in the SS research area [7, 12]. Several contributions have focussed on eigenvalue-based sensing techniques for large scale CR networks using RMT [6, 7, 12-14]. In addition, different Signal Condition Number (SCN) based techniques such as asymptotic [12], semi-asymptotic [6] and ratio based techniques [14] have been proposed in the literature. The SS techniques based on Marchenko-Pastur (MP) deterministic bounds have been proposed in [12]. Furthermore, SS techniques using the TracyWidom distribution and the Tracy-Widom Curtiss distribution have been proposed in [6] and [14] respectively.

Most SS related contributions focus on a dual hypothesis test to decide the presence or absence of primary on-going transmissions. In practice, it may be the case that a spectrum resource is completely left unused within a sufficiently large network coverage area. The optimal exploitation of the spectrum holes in such a scenario depends on the maximally acceptable coverage area of secondary transmission which protects the primary rate [9]. If the $\mathrm{CR}$ is able to estimate the SNR of the primary signal, it can dynamically adapt its coverage area using underlay techniques. Despite its important applications, only a few contributions in the literature address the SNR estimation problem in the context of a CR $[15,16]$. In [15], an SNR estimation method has been proposed for UltraWide Band (UWB) CR systems using computer simulations and this method is specific only for multi-band orthogonal frequency division multiplexing based systems. In [16], a pseudo Bit Error Rate (BER) based SNR estimation has been proposed for Energy Detection (ED) scheme. Furthermore, in practical scenarios, the fading channel may be correlated due to various causes such as insufficient scattering in the propagation path and antenna mutual coupling $[17,18]$. In this context, the contributions in $[19,20]$ studied the sensing performance of an energy detector when multiple antennas are correlated.

Taking the above into account, exploring efficient SNR estimation techniques in the presence of channel correlation is 
an important research challenge. In this context, we consider the PU SNR estimation problem in the presence of channel correlation. For this purpose, the asymptotic eigenvalue probability distribution function (a.e.p.d.f) under signal plus noise hypothesis is needed but still missing in the literature. In our previous work [21], the effect of noise correlation on eigenvalue-based SS techniques has been studied and the a.e.p.d.f of the received signal's covariance matrix has been derived in the presence of correlated noise. In this paper, we derive the expression for the a.e.p.d.f. of the received signal's covariance matrix in the presence of channel correlation using RMT. This is the main contribution of this paper. Furthermore, we use the derived a.e.p.d.f. to estimate the PU SNR in presence of channel correlation based on the maximum eigenvalue. Moreover, the performance of the proposed technique is evaluated in terms of normalized Mean Square Error (MSE).

The remainder of this paper is structured as follows: Section II describes the considered signal model and further presents channel correlation modeling used in this paper. Section III presents the channel correlation analysis using RMT and further proposes the eigenvalue-based SNR estimation technique using the derived a.e.p.d.f for received signal's covariance matrix. Section IV studies the performance of the proposed SNR estimation technique with numerical simulations. Section V concludes the paper. The appendix includes some preliminaries on random matrix transforms.

\section{A. Notation}

Throughout this paper, $\mathbb{E}[\cdot]$ denotes expectation, $(\cdot)^{T}$ and $(\cdot)^{H}$ denote the transpose and the conjugate transpose respectively, $(\cdot)^{*}$ represents the complex conjugate, I denotes the identity matrix, $\mathcal{S}_{\mathbf{X}}$ represents the Stieltjes transform of $\mathbf{X}, \mathcal{R}_{\mathbf{X}}$ represents the $\mathrm{R}$ transform and $\Sigma_{\mathbf{X}}$ represents the $\Sigma$ transform [8].

\section{Signal Model}

Let us consider a single cognitive user and a single PU for simplicity of analysis. Let $N$ be the number of samples analyzed by the cognitive user for the decision process and $\tau$ the sensing duration. Let $M$ be the number of receive dimensions in the cognitive receiver. From a system-model point of view, this factor can be considered to be the number of antennas in a multiantenna-based CR receiver and the number of oversampled branches in an oversampling-based sensing model as considered in $[6,7,21]$. The signal model presented in this paper is generic and can be applicable to most multidimensional scenarios.

Let us denote the hypotheses of the presence and absence of the PU signal by $\mathbb{H}_{1}$ and $\mathbb{H}_{0}$ respectively. A binary hypothesis testing problem for the $k$ th receive dimension, $k=1, \ldots, M$, can be written as:

$$
\begin{aligned}
& \mathbb{H}_{0}: y_{k}(i)=z_{k}(i) \\
& \text { PU absent } \\
& \mathbb{H}_{1}: y_{k}(i)=\hat{h}_{k}(i) s(i)+z_{k}(i), \quad \text { PU present }
\end{aligned}
$$

where $y_{k}(i)$ is the signal observed by the $k$ th receiving dimension at the $i$ th instant, $i=1,2, . ., N$ denotes the sampling instant, $s(i)$ is the PU signal at the $i$ th instant, whose SNR is to be estimated, $\hat{h}_{k}(i)$ is the amplitude gain of the channel for the $k$ th receive dimension at the $i$ th instant, and $z_{k}(i)$ denotes the white noise for the $k$ th receive dimension at the $i$ th instant. For our analysis, we assume that transmitted symbols are independent and identically distributed (i.i.d.) complex circularly symmetric (c.c.s.) Gaussian symbols, the noise samples are independent across both dimensions. For channel correlation analysis, we assume channel coefficients of $M \times N$ channel matrix $\hat{\mathbf{H}}$ to be i.i.d. across samples and correlated across the receive dimensions as reflected in the Section II-A. The sensing duration $(\tau)$ and symbol interval $\left(T_{s}\right)$ may not be the same depending on the signal bandwidth and sampling rate used at the $\mathrm{CR}$ receiver. Based on the relation between $\tau$ and $T_{s}$, we consider the following signal models under the $\mathbb{H}_{1}$ hypothesis.

Case 1: In this case, we consider that the transmitted symbol remains constant during the sensing period. This case may result when the sampling rate at the receiver is much higher than the transmitted symbol rate. The $M \times N$ received signal matrix $\mathbf{Y}$ in this case can be written as: $\mathbf{Y}=\sqrt{p} \hat{\mathbf{H}} s+\mathbf{Z}$, where $s$ is a constant transmitted symbol, $p$ is the power of transmitted symbol and $\mathbf{Z} \triangleq\left[\mathbf{z}_{1}^{T}, \mathbf{z}_{2}^{T}, \ldots, \mathbf{z}_{M}^{T}\right]^{T}$, with $\mathbf{z}_{m} \triangleq\left[\begin{array}{llll}z_{m}(1) & z_{m}(2) & \ldots & z_{m}(N)\end{array}\right]$. The $M \times N$ correlated channel matrix $\hat{\mathbf{H}} \triangleq\left[\hat{\mathbf{h}}_{1}^{T}, \hat{\mathbf{h}}_{2}^{T}, \ldots, \hat{\mathbf{h}}_{M}^{T}\right]^{T}$, with $\hat{\mathbf{h}}_{m} \triangleq$ $\left[\begin{array}{llll}\hat{h}_{m}(1) & \hat{h}_{m}(2) & \ldots & \hat{h}_{m}(N)\end{array}\right]$ with $m=1,2, \ldots, M$. Since we assume normalized noise variance, $\mathrm{SNR} \equiv p$. In this case, the covariance of the transmitted signal can be written as: $R_{s}=\mathbb{E}\left[s^{2}\right]=1$.

Case 2: In this case, each column of $\mathbf{Y}$, includes the samples for a single symbol. $\mathbf{Y}=\sqrt{p} \hat{\mathbf{H}} \mathbf{S}_{d}+\mathbf{Z}$, where $\mathbf{S}_{d}$ is the diagonal transmitted signal matrix of dimension $N \times N$ with diagonal $\mathbf{s}=[s(1) \ldots s(N)]$. In this case, the covariance matrix of the transmitted signal becomes

$$
\mathbf{R}_{\mathbf{S}}=\mathbb{E}\left[\mathbf{S}_{d} \mathbf{S}_{d}^{H}\right]=\left[\begin{array}{cccc}
\mathbb{E}\left[s^{2}(1)\right] & 0 & \cdots & 0 \\
0 & \mathbb{E}\left[s^{2}(2)\right] & \cdots & 0 \\
& & \ddots & \\
0 & 0 & \cdots & \mathbb{E}\left[s^{2}(N)\right]
\end{array}\right]=\mathbf{I} .
$$

assuming that for each sample, we get an i.i.d. c.c.s. symbol with $\mathbb{E}\left[s^{2}\right]=1$.

The received signal matrix $\mathbf{Y}$ in both cases can be written in the following form.

$$
\mathbf{Y}=\left[\begin{array}{c}
\mathbf{y}_{1} \\
\mathbf{y}_{2} \\
\vdots \\
\mathbf{y}_{M}
\end{array}\right]=\left[\begin{array}{cccc}
y_{1}(1) & y_{1}(2) & \ldots & y_{1}(N) \\
y_{2}(1) & y_{2}(2) & \ldots & y_{2}(N) \\
\vdots & \vdots & \ddots & \vdots \\
y_{M}(1) & y_{M}(2) & \ldots & y_{M}(N)
\end{array}\right]
$$

The received signal $\mathbf{Y}$ can be further written as:

$$
\mathbf{Y}= \begin{cases}\sqrt{p} \hat{\mathbf{H}} s+\mathbf{Z}, & \text { Case } 1 \\ \sqrt{p} \hat{\mathbf{H}} \mathbf{S}_{d}+\mathbf{Z}, & \text { Case } 2\end{cases}
$$

where $\mathbf{Z} \sim \mathcal{C N}(0, \mathbf{I})$ is the additive white Gaussian noise. Assuming that the source signal is independent from the white noise, the covariance matrix of received signal $\mathbf{R}_{\mathbf{Y}}$ can be 
calculated as $[6]$ :

$$
\begin{aligned}
\mathbf{R}_{\mathbf{Y}} & =\mathbb{E}\left[\mathbf{Y} \mathbf{Y}^{H}\right]=\mathbb{E}\left[(\sqrt{p} \hat{\mathbf{H}} \mathbf{S})(\sqrt{p} \hat{\mathbf{H}} \mathbf{S})^{H}\right]+\mathbb{E}\left[\mathbf{Z} \mathbf{Z}^{H}\right] \\
& =p \mathbb{E}\left[\hat{\mathbf{H}} \hat{\mathbf{H}}^{H}\right]+\mathbf{R}_{\mathbf{Z}}
\end{aligned}
$$

where $\mathbf{R}_{\mathbf{Z}}=\mathbb{E}\left[\mathbf{Z Z}^{H}\right]$. Let us define sample covariance matrices of the received signal and noise as: $\hat{\mathbf{R}}_{\mathbf{Y}}(N)=\frac{1}{N} \mathbf{Y} \mathbf{Y}^{H}$ and $\hat{\mathbf{R}}_{\mathbf{Z}}(N)=\frac{1}{N} \mathbf{Z} \mathbf{Z}^{H}$.

\section{A. Channel Correlation Modeling}

The channel correlation in MIMO/Single Input Multiple Output (SIMO) systems depends on propagation [18] and antenna mutual coupling [17]. In a wireless multipath environment, the channels are not always independent from each other but can be correlated due to poor scattering in the propagation path. This type of correlation can be referred as spatial correlation. Channel correlation also arises due to mutual coupling between the transmit and/or receive antenna elements $[17,22]$. In the transmitter and receive antenna arrays, antenna mutual coupling causes the signals to be coupled with the neighbouring antennas.

For modeling channel correlation, we use the one-sided correlation model as in [11]. In this paper, we focus on the correlation across channel receive dimensions and not on the temporal correlation. We model the channel as:

$$
\hat{\mathbf{H}}=\boldsymbol{\Phi}^{1 / 2} \mathbf{H},
$$

where $\mathbf{H}$ is an $M \times N$ matrix with c.c.s. i.i.d. Gaussian entries with zero mean and unit variance and $\boldsymbol{\Phi}^{1 / 2} \boldsymbol{\Phi}^{1 / 2}=$ $\boldsymbol{\Phi}=\mathbb{E}\left[\hat{\mathbf{H}} \hat{\mathbf{H}}^{H}\right]$. It can be noted that since $\mathbf{H} \sim$ $\mathcal{C N}(0, \mathbf{I}), \mathbf{H H}^{H}$ follows uncorrelated Wishart distribution i.e., $\mathbf{H H}^{H} \sim \mathcal{W}_{M}\left(\mathbf{R}_{\mathbf{H}}, N\right)$. To ensure that $\boldsymbol{\Phi}$ does not affect the channel power, we consider the following normalization: $(1 / M) \operatorname{trace}\{\boldsymbol{\Phi}\}=1$, where each component of $\boldsymbol{\Phi}$ is modeled with an exponential covariance model given by [10];

$$
\phi_{i j}= \begin{cases}\varsigma^{(j-i)}, & i \leq j \\ \left(\varsigma^{(i-j)}\right)^{*}, & i>j\end{cases}
$$

where $\phi_{i j}$ is the $(i, j)$-th element of $\boldsymbol{\Phi}$ and $\varsigma \in \mathbb{C}$ is the correlation coefficient with $|\varsigma| \leq 1$.

\section{Channel Correlation Analysis Using RMT}

For the purpose of completeness in this paper, we state the following RMT theorem which is going to be used in our analysis.

Theorem 3.1: [11] Let $\Theta$ be a positive definite matrix which is normalized as: $(1 / M) \operatorname{trace}\{\boldsymbol{\Theta}\}=1$, and whose asymptotic spectrum has the p.d.f.

$$
f_{\Theta}(\lambda)=\frac{1}{2 \pi \mu \lambda^{2}} \sqrt{\left(\frac{\lambda}{\sigma_{1}}-1\right)\left(1-\frac{\lambda}{\sigma_{2}}\right)}
$$

with $\sigma_{1} \leq \lambda \leq \sigma_{2}$ and $\mu=\frac{\left(\sqrt{\sigma_{2}}-\sqrt{\sigma_{1}}\right)^{2}}{4 \sigma_{1} \sigma_{2}}$. If $\mathbf{F}$ is an $M \times N$ standard complex Gaussian matrix and $\frac{1}{N} \mathbf{F F}^{H}$ follows the MP distribution [8], then as $M, N \rightarrow \infty$ with $\frac{N}{M} \rightarrow \beta$, the asymptotic eigenvalue distribution of $\mathbf{W}=\boldsymbol{\Theta}^{1 / 2} \mathbf{F F}{ }^{H} \boldsymbol{\Theta}^{1 / 2}$ has the following p.d.f.

$$
f_{\mathbf{W}}(\lambda)=(1-\beta)^{+} \delta(\lambda)+\frac{\sqrt{(\lambda-\tilde{a})^{+}(\tilde{b}-\lambda)^{+}}}{2 \pi \lambda(1+\lambda \mu)},
$$

where

$$
\begin{aligned}
& \tilde{a}=1+\beta+2 \mu \beta-2 \sqrt{\beta} \sqrt{(1+\mu)(1+\mu \beta)} \\
& \tilde{b}=1+\beta+2 \mu \beta+2 \sqrt{\beta} \sqrt{(1+\mu)(1+\mu \beta)}
\end{aligned}
$$

The parameters $\tilde{a}$ and $\tilde{b}$ correspond to $\lambda_{\min }^{\infty}$ and $\lambda_{\max }^{\infty}$ respectively and the ratio $\tilde{b} / \tilde{a}$ defines the SCN of $\mathbf{W}$.

The above theorem is applicable for channel covariance matrix $\boldsymbol{\Phi}$. The eigenvalue spread of $\boldsymbol{\Phi}$ is related to the degree of channel covariance i.e., a zero eigenvalue spread corresponds to a zero-covariance model and higher spreads are associated with higher covariance models. In (9), the parameter $\mu$ controls the degree of covariance and varies the support of the distribution i.e., for $\mu=0, \tilde{a}=a$ and $\tilde{b}=b$, where $a=(1-\sqrt{\beta})^{2}$ and $b=(1+\sqrt{\beta})^{2}$ are the supports of the MP distribution [8]. For the exponential covariance model as stated in [11], the parameter $\mu$ is related to the correlation coefficient $\varsigma$ with the following relation: $\mu=\frac{\varsigma^{2}}{1-\varsigma^{2}}$. Furthermore, the SCN is related to $\varsigma$ with the relation $\mathrm{SCN}=\frac{1+\varsigma}{1-\varsigma}$. In practical scenarios, the value of $\mu$ can be determined using channel estimation techniques. The received signal matrix can also be expressed as:

$$
\mathbf{Y}=\sqrt{p} \boldsymbol{\Phi}^{\frac{1}{2}} \mathbf{H} \mathbf{S}_{d}+\mathbf{Z}
$$

Assuming that signal and noise are independent, for very large value of $N$, the sample covariance matrix of received signal under considered scenario in the presence of channel correlation can be approximated as [6]:

$$
\lim _{N \rightarrow \infty} \hat{\mathbf{R}}_{\mathbf{Y}}(N) \approx p \boldsymbol{\Phi}^{\frac{1}{2}} \mathbf{H} \mathbf{H}^{H} \boldsymbol{\Phi}^{\frac{1}{2}}+\hat{\mathbf{R}}_{\mathbf{Z}}
$$

Due to noncommutative nature of random matrices, it's not straightforward to calculate the eigenvalue distributions of the received signal $\mathbf{Y}$ by knowing the eigenvalue distribution of covariance matrices of $\boldsymbol{\Phi}, \mathbf{H}$ and $\mathbf{Z}$ in (12). In the following section, we provide the methodology for calculating the a.e.p.d.f. of the received signal's covariance matrix which is then used for SNR estimation in the presence of channel correlation.

\section{A. Methodology}

The a.e.p.d.f. of the received signal's covariance matrix under $\mathbb{H}_{1}$ hypothesis can be used to estimate the PU SNR by a CR. To calculate the a.e.p.d.f. of received signal's sample covariance matrix i.e., $\hat{\mathbf{R}}_{\mathbf{Y}}(N)$, we need to know the Stieltjes transform of the asymptotic density function of the $\hat{\mathbf{R}}_{\mathbf{Y}}(N)$. Using free probability analysis, the asymptotic spectrum of the sum or product can be obtained from the individual asymptotic spectra without involving the structure of the eigenvectors of the matrices [8] under an asymptotic freeness condition. The asymptotic eigenvalue distribution of $\mathbf{Y}$ in our context can be 
obtained by applying $\Sigma$ and R transforms [8]. In (12), since $\boldsymbol{\Phi}$ is a deterministic matrix and $\mathbf{H} \mathbf{H}^{H}$ is a Wishart random matrix, they are asymptotically free (see Example 2.34, [8]). As a result, the combined a.e.p.d.f. of the term $p \boldsymbol{\Phi}^{\frac{1}{2}} \mathbf{H} \mathbf{H}^{H} \boldsymbol{\Phi}^{\frac{1}{2}}$ in (12) can be obtained by applying multiplicative free convolution property of $\Sigma$ transform in the following way [23].

$$
\Sigma_{p \hat{\mathbf{R}}_{\hat{\mathbf{H}}}}(z)=\Sigma_{\boldsymbol{\Phi}}(z) \cdot \Sigma_{p \hat{\mathbf{R}}_{\mathbf{H}}}(z),
$$

where $\Sigma_{\boldsymbol{\Phi}}$ and $\Sigma_{p \hat{\mathbf{R}}_{\mathbf{H}}}$ are the $\Sigma$ transforms of the densities of eigenvalues of $\boldsymbol{\Phi}$ and $p \hat{\mathbf{R}}_{\mathbf{H}}$ respectively. Since $\boldsymbol{\Phi}$ is a square matrix, $\boldsymbol{\Phi}^{1 / 2} \mathbf{H} \mathbf{H}^{H} \boldsymbol{\Phi}^{1 / 2}$ and $\boldsymbol{\Phi} \mathbf{H} \mathbf{H}^{H}$ have identical eigenvalues [8]. Furthermore, $\mathbf{\Phi} \mathbf{H} \mathbf{H}^{H}$ and $\mathbf{Z} \mathbf{Z}^{H}$ are independent and $\mathbf{Z} \mathbf{Z}^{H}$ is a Wishart matrix. As a result, $\mathbf{\Phi} \mathbf{H} \mathbf{H}^{H}$ and $\mathbf{Z} \mathbf{Z}^{H}$ are asymptotically free due to their unitarily invariance property [9]. Then the combined $R$ transform of $\hat{\mathbf{R}}_{\mathbf{Y}}$ can be found from the $\mathrm{R}$ transforms of $\hat{\mathbf{R}}_{\mathbf{Z}}$ and $p \hat{\mathbf{R}}_{\hat{\mathbf{H}}}$ using additive free convolution property of $\mathrm{R}$ transform in the following way [24].

$$
\mathcal{R}_{\hat{\mathbf{R}}_{\mathbf{Y}}}(z)=\mathcal{R}_{\hat{\mathbf{R}}_{\mathbf{Z}}}(z)+\mathcal{R}_{p \hat{\mathbf{R}}_{\hat{\mathbf{H}}}}(z) .
$$

Since $\hat{\mathbf{R}}_{\mathbf{Z}}$ follows MP law, its $\mathrm{R}$ transform can be written as the $\mathrm{R}$ transform of MP law and is given by (25). Using the transformations included in the preliminaries, we get the Stieltjes transform of $\hat{\mathbf{R}}_{\mathbf{Y}}$ as described in the next subsection. The a.e.p.d.f. of $\mathbf{Y}$ is then obtained by determining the imaginary part of the Stieltjes transform $\mathcal{S}_{\hat{\mathbf{R}}_{\mathbf{Y}}}$ for real arguments in the following way.

$$
f(x)=\lim _{y \rightarrow 0^{+}} \frac{1}{\pi} \operatorname{Im}\left\{\mathcal{S}_{\hat{\mathbf{R}}_{\mathbf{Y}}}(x+j y)\right\} .
$$

\section{B. Main Result}

For the purpose of completeness, we present the Stieltjes transform for an uncorrelated channel and white noise case from [21]. The Stieltjes transform $\mathcal{S}_{\hat{\mathbf{R}}_{\mathbf{Y}}}$ of the asymptotic distribution of $\frac{1}{N} \mathbf{Y} \mathbf{Y}^{H}$ for this case can be obtained for any $z \in \mathbb{C}$ by solving the following cubic polymonial [21].

$$
\begin{aligned}
& c_{0}=1, \\
& c_{1}=(1-\beta)(1-p)+z, \\
& c_{2}=p(-2 \beta+z+1)+z, \\
& c_{3}=p z,
\end{aligned}
$$

where $c_{n}$ is the $n$th order coefficient of the polymonial, $\beta=\frac{N}{M}$ and $p$ is the SNR of the transmitted PU signal.

Theorem 3.2: The Stieltjes transform $\mathcal{S}_{\hat{\mathbf{R}}_{\mathbf{Y}}}$ of the asymptotic distribution of eigenvalues of $\frac{1}{N} \mathbf{Y} \mathbf{Y}^{H}$ where $\mathbf{Y}=$ $\hat{\mathbf{H}} \mathbf{S}_{d}+\mathbf{Z}$ can be obtained for any $z \in \mathbb{C}$ by solving a quartic polymonial with the following coefficients

$$
\begin{aligned}
& c_{0}=1+\mu, \\
& c_{1}=2 \mu(z+1-\beta)-\beta(p+1)+p+z+2, \\
& c_{2}=p(z-3 \beta+2)+\beta(\mu(\beta-2 z-2)-1) \\
& +\mu(z(z+\mu)+1)+2 z, \\
& c_{3}=-2 p(\beta-z)+2 z \mu(1-\beta+z)+p+z, \\
& c_{4}=z(p+\mu z),
\end{aligned}
$$

where the parameter $\mu$ defines the degree of covariance of the channel covariance matrix $\boldsymbol{\Phi}$.

Proof: Assuming that signal and noise are uncorrelated to each other, for very large value of $N$, the following approximation can be written for correlated channel and white noise scenario [6].

$$
\lim _{N \rightarrow \infty} \hat{\mathbf{R}}_{\mathbf{Y}}(N) \approx p \hat{\mathbf{H}} \hat{\mathbf{H}}^{H}+\hat{\mathbf{R}}_{\mathbf{Z}}(N) .
$$

The Stieltjes transform of $\hat{\mathbf{R}}_{\hat{\mathbf{H}}}=\frac{1}{N} \hat{\mathbf{H}} \hat{\mathbf{H}}^{H}$ can be written as [11]:

$$
\mathcal{S}_{\hat{\mathbf{R}}_{\hat{\mathbf{H}}}}(z)=\frac{z+2 z \mu+1-\beta+\sqrt{[z-(1+\beta)]^{2}-4 \beta(1+\mu z)}}{2 z(1+\mu z)} .
$$

The $\mathrm{R}$ transform of $\hat{\mathbf{R}}_{\hat{\mathbf{H}}}$ can be found by using (24). The $\mathrm{R}$ transform of $p \hat{\mathbf{R}}_{\hat{\mathbf{H}}}$ can be found by using (26) and can be written as:

$$
\mathcal{R}_{p \hat{\mathbf{R}}_{\hat{\mathbf{H}}}}(z)=-\frac{p}{2} \frac{\left(p z-1+\sqrt{\left(p^{2} z^{2}-2 p z+1-4 \mu \beta p z\right)}\right)}{\mu p z} .
$$

Then the combined $\mathrm{R}$ transform for $\hat{\mathbf{R}}_{\mathbf{Y}}$ can be written as:

$$
\begin{aligned}
\mathcal{R}_{\hat{\mathbf{R}}_{\mathbf{Y}}}(z) & =-\frac{p}{2} \frac{\left(p z-1+\sqrt{\left(p^{2} z^{2}-2 p z+1-4 \mu \beta p z\right)}\right)}{\mu p z} \\
& +\frac{\beta}{(1-z)} .
\end{aligned}
$$

The inverse Stieltjes transform can be obtained by applying (21) on (24) and then the Stieltjes transform can be obtained by solving the quartic polymonial given by (17).

The theoretical analysis for SNR estimation in the presence of both noise and channel correlation can be found in [25].

\section{SNR Estimation Method}

The Stieltjes transforms of $\hat{\mathbf{R}}_{\mathbf{Y}}$ for uncorrelated channel plus white noise and correlated channel plus white noise cases are obtained solving polymonials (16) and (17) respectively. Then the supports for the a.e.p.d.f. of $\hat{\mathbf{R}}_{\mathbf{Y}}$ for the corresponding case is calculated using (15). Since we know the value of $\beta$ and we can measure the value of $\varsigma$, we can estimate the value of $p$ by sensing the maximum eigenvalue of $\hat{\mathbf{R}}_{\mathbf{Y}}$. The value of $\varsigma$ for correlated channel can be found with channel estimation methods such as [26]. Furthermore, it can be noted that the parameters $\beta$ and $\varsigma$ are assumed as operating parameters of the CR sensing module. For convenience, lookup table (Table I) is provided in Section IV in order to estimate the PU SNR. We consider the following two cases: (i) uncorrelated channel plus white noise, and (ii) correlated channel plus white noise. In Table I, we include the maximum eigenvalues of received signal's covariance matrix for these cases for different values of SNR and $\beta$. With the help of this table, we can estimate SNR of the PU signal based on the maximum eigenvalue of received signal's covariance matrix.

\section{NUMERICAL RESUlTS}

In this section, we evaluate the performance of the proposed SNR estimation method in the presence of correlation using 
normalized MSE. The normalized MSE is considered as a parameter to characterize the performance of the proposed SNR estimation technique and is defined as:

$$
\mathrm{MSE}=\frac{\mathbb{E}\left[(\hat{p}-p)^{2}\right]}{p^{2}},
$$

where $\hat{p}$ is the estimated SNR with the proposed method and $p$ is the actual SNR.

Figure 1 shows the theoretical and simulated eigenvalue distribution of the received signal's covariance matrix for correlated channel and white noise scenario. The histograms of the eigenvalues were created by accumulating the eigenvalues over $10^{3}$ realizations. The theoretical result was obtained by evaluating the polynomial (17) with the help of a mathematical software. From the figure, it can be noted that the theoretical and simulated eigenvalue distributions match well. Furthermore, it has been noted that eigenvalue distributions differ depending on the values of the parameters SCN, SNR and $\beta$.

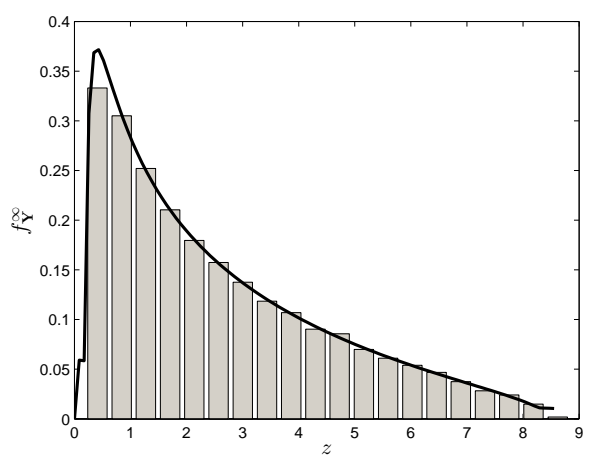

Fig. 1: Theoretical and simulated eigenvalue distribution of the received signal's covariance matrix for correlated channel and white noise scenario i.e., $\mathbf{Y}=\left(\hat{\mathbf{H}} \mathbf{S}_{d}+\mathbf{Z}\right)(\mathrm{SCN}=4, \mathrm{SNR}=2 \mathrm{~dB}, \beta=1, N=100)$

Table I shows the lookup table for different values of SCN of the channel covariance matrix and it is obtained using the methodology described under Section III-C. This table can be used to estimate the SNR of the PU signal based on the values of $\mathrm{SCN}$ and $\beta$ for two different cases shown in the table. For example, if the value of $\mathrm{SCN}$ is $2, \beta$ is 1 and the maximum eigenvalue of sample covariance matrix of received signal i.e., $\frac{1}{N} \mathbf{Y} \mathbf{Y}^{H}$ is 5.65 in case II, we can then estimate that SNR of the PU signal is $0 \mathrm{~dB}$ and intermediate values can be calculated through interpolation. Figure 2 shows the maximum eigenvalue versus SNR for different cases $(\beta=1$, $N=100)$. From the figure, it can be noted that the maximum eigenvalue increases with the SNR for both correlated and uncorrelated channel cases with the higher rate in the high SNR region. Furthermore, the rate of increase is higher for correlated channel case than for uncorrelated channel case. It can be further noted that the maximum eigenvalue increases with the value of SCN and the effect of channel correlation is more dominant at high SNR values.

Figure 3 shows the normalized MSE versus SNR for the considered cases with the simulation parameters $(\beta=1$, $\mathrm{SCN}=4$ and $N=100)$. In this simulation setting, we generate instances of $\mathbf{Y}$ according to the considered case and we evaluate the MSE performance with (22) considering the corresponding case from the table. From the figure, it can be noted that the value of normalized MSE decreases with the increase in SNR for both cases. It can be further noted that the SNR for both cases can be reliably estimated with almost $0.2 \%$ normalized MSE after $0 \mathrm{~dB}$. Moreover, it can be noted that at lower SNR values, case II has lower normalized MSE than case I and at higher values of SNR (after SNR value of $0 \mathrm{~dB}$ ), case II has higher normalized MSE than the case I. It can be concluded that channel correlation effect is dominant at higher SNR values in comparison to the uncorrelated case. The detailed description on the combined effect of noise and correlation on the SNR estimation performance of the proposed techniques can be found in [21].

TABLE I: Lookup table for SNR Estimation

\begin{tabular}{l|c|c|c|c}
$\mathbf{S C N}$ & $\beta=N / M$ & $\mathbf{S N R}(\mathrm{dB})$ & $\begin{array}{c}\mathbf{C a s e}_{\mathbf{I}} \\
\lambda_{\max }\left(\mathbf{H} \mathbf{S}_{d}+\mathbf{Z}\right)\end{array}$ & $\begin{array}{c}\text { Case II } \\
\lambda_{\max }\left(\hat{\mathbf{H S}}_{d}+\mathbf{Z}\right)\end{array}$ \\
\hline 2 & 1 & 5 & 13.18 & 13.51 \\
2 & 1 & 4 & 10.77 & 11.03 \\
2 & 1 & 2 & 7.45 & 7.58 \\
2 & 1 & 0 & 5.59 & 5.65 \\
2 & 1 & -2 & 4.70 & 4.71 \\
2 & 1 & -4 & 4.29 & 4.29 \\
2 & 1 & -6 & 4.08 & 4.08 \\
\hline 4 & 1 & 5 & 13.18 & 14.55 \\
4 & 1 & 4 & 10.77 & 8.05 \\
4 & 1 & 2 & 7.45 & 5.86 \\
4 & 1 & 0 & 5.59 & 4.78 \\
4 & 1 & -2 & 4.70 & 4.31 \\
4 & 1 & -4 & 4.29 & 4.08 \\
4 & 1 & -6 & 4.08 & \\
\hline
\end{tabular}

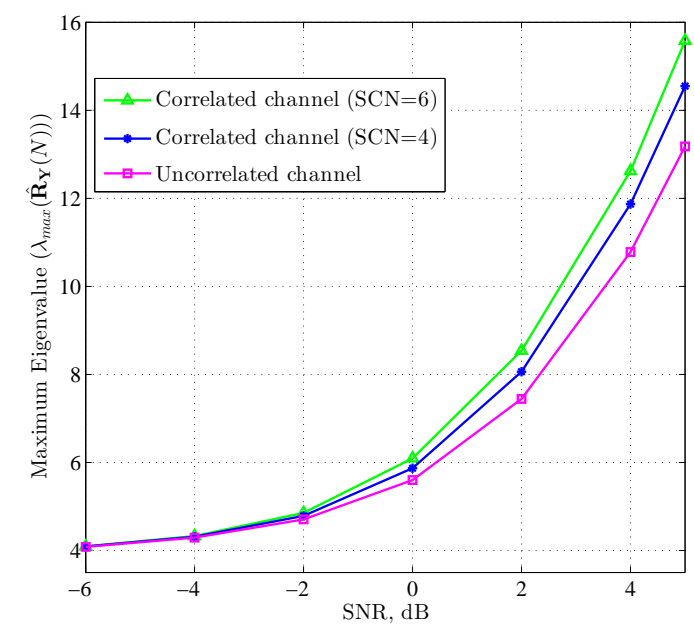

Fig. 2: Maximum eigenvalue versus SNR for uncorrelated and correlated channel scenarios $(\beta=1, N=100)$

\section{CONCLUSION}

In this paper, the PU SNR estimation problem has been considered in the context of a CR. The motivation for this problem comes from the fact that with the knowledge of the PU SNR, suitable underlay strategies can be implemented at the CRs in order to allow the coexistence of the primary and secondary systems. The theoretical expression for a.e.p.d.f of the received signal's covariance matrix has been derived in presence of channel correlation. Moreover, an SNR estimation technique based on the maximum eigenvalue of the received signal's covariance matrix has been presented in order to estimate the PU SNR reliably in the presence of channel correlation. The 


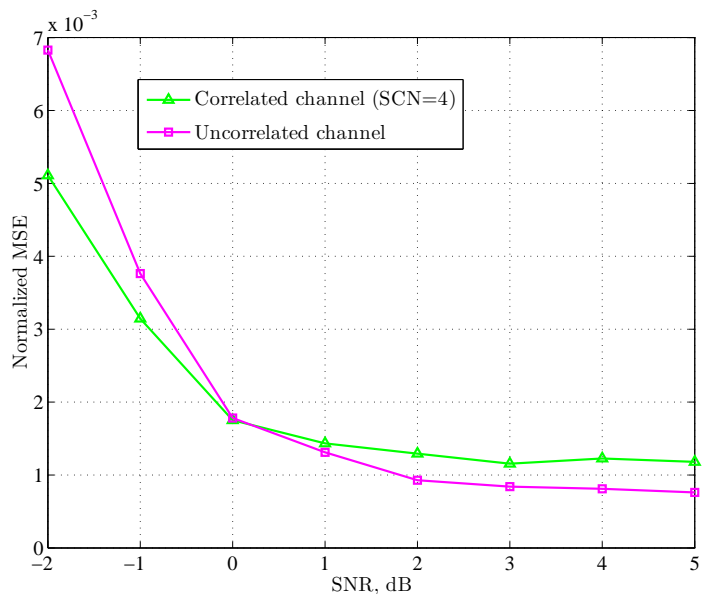

Fig. 3: Normalized MSE versus SNR for uncorrelated and correlated channel scenarios $(\beta=1, N=100)$

performance of the proposed technique has been evaluated in terms of normalized MSE. It can be concluded that the PU SNR can be reliably estimated by a CR in the presence of channel correlation using the proposed technique.

\section{APPENDIX}

\section{Random Matrix Theory Preliminaries}

Let $F_{\mathbf{X}}(x)$ be the eigenvalue probability density function of a matrix $\mathbf{X}$.

Theorem 5.1: The Stieltjes transform $\mathcal{S}_{\mathbf{X}}(z)$ of a positive semidefinite matrix $\mathbf{X}$ is defined by [8]:

$$
\mathcal{S}_{\mathbf{X}}(z)=\mathbb{E}\left[\frac{1}{\mathbf{X}-z}\right]=\int_{-\infty}^{\infty} \frac{1}{\lambda-z} d F_{\mathbf{X}}(\lambda) .
$$

Theorem 5.2: The $\mathrm{R}$ transform is related to the inverse of Stieltjes transform as [8]:

$$
\mathcal{R}_{\mathbf{X}}(z)=\mathcal{S}_{\mathbf{X}}^{-1}(-z)-\frac{1}{z} .
$$

Theorem 5.3: For a Wishart random matrix $\mathbf{X}$, the $\mathrm{R}$ transform of the density of eigenvalues of $\mathbf{X}$ is defined as [8]:

$$
\mathcal{R}_{\mathbf{X}}(z)=\frac{\beta}{1-z} .
$$

For any $a>0$,

$$
\mathcal{R}_{a \mathbf{X}}=a \mathcal{R}_{\mathbf{X}}(a z)
$$

Theorem 5.4: For a Wishart random matrix $\mathbf{X}$, the $\Sigma$ transform of the density of eigenvalues of $\mathbf{X}$ is defined as [8]:

$$
\Sigma_{\mathbf{X}}(z)=\frac{1}{z+\beta} .
$$

\section{ACKNOWLEDGEMENT}

This work was supported by the National Research Fund, Luxembourg under AFR (Aids Training-Research) grant for $\mathrm{PhD}$ project (Reference 3069102) and the CORE project "CO2SAT: Cooperative and Cognitive Architectures for Satellite Networks".

\section{REFERENCES}

[1] A. Goldsmith, S. Jafar, I. Maric, and S. Srinivasa, "Breaking spectrum gridlock with cognitive radios: An information theoretic perspective," Proc. IEEE, vol. 97, no. 5, pp. 894 -914, May 2009.
[2] S. K. Sharma, S. Chatzinotas, and B. Ottersten, "Satellite cognitive communications: Interference modeling and techniques selection," in 6th ASMS/SPSC Conf., Sept. 2012, pp. 111-118.

[3] T. Yucek and H. Arslan, "A survey of spectrum sensing algorithms for cognitive radio applications," IEEE Commun. Surveys Tut., vol. 11, no. 1, pp. $116-130$, quarter 2009.

[4] S. K. Sharma, S. Chatzinotas, and B. Ottersten, "Exploiting polarization for spectrum sensing in cognitive SatComs," in Proc. 7th Int. Conf. CROWNCOM, June 2012.

[5] S. K. Sharma, S. Chatzinotas, and B. Ottersten, "Spectrum sensing in dual polarized fading channels for cognitive SatComs," in Proc. IEEE GLOBECOM, Dec. 2012.

[6] Y. Zeng and Y. chang Liang, "Eigenvalue-based spectrum sensing algorithms for cognitive radio," IEEE Trans. Commun., vol. 57, no. 6, pp. $1784-1793$, June 2009.

[7] W. Zhang, G. Abreu, M. Inamori, and Y. Sanada, "Spectrum sensing algorithms via finite random matrices," IEEE Trans. Commun., vol. 60, no. 1 , pp. $164-175$, Jan. 2012.

[8] A. M. Tulino and S. Verdu, "Random matrix theory and wireless communications," Foundations and Trends in Commun. and Inf. Th., vol. 1, no. 1, pp. 1-182, 2004.

[9] R. Couillet and M. Debbah, Random Matrix Methods for Wireless Communications, 1st ed. Cambridge University Press, 2011.

[10] S. Chatzinotas, M. Imran, and R. Hoshyar, "On the multicell processing capacity of the cellular MIMO uplink channel in correlated Rayleigh fading environment," IEEE Trans. Wireless Commun., vol. 8, no. 7, pp. $3704-3715$, July 2009.

[11] X. Mestre, J. Fonollosa, and A. Pages-Zamora, "Capacity of MIMO channels: asymptotic evaluation under correlated fading," IEEE J. Sel. Areas Commun., vol. 21, no. 5, pp. 829 - 838, June 2003.

[12] L. Cardoso, and et al, "Cooperative spectrum sensing using random matrix theory," in 3rd Int. Symp. Wireless Pervasive Comp., May 2008, pp. $334-338$.

[13] K. Hassan, and et al, "Predicted eigenvalue threshold based spectrum sensing with correlated multiple-antennas," in IEEE 75th Veh. Technol. Conf., May 2012, pp. $1-5$.

[14] F. Penna, R. Garello, and M. Spirito, "Cooperative spectrum sensing based on the limiting eigenvalue ratio distribution in Wishart matrices," IEEE Commun. Letters, vol. 13, no. 7, pp. 507 -509, July 2009.

[15] M. Fujii and Y. Watanabe, "A study on SNR estimation for cognitive radio," in IEEE Int. Conf. UWB, Sept. 2012, pp. $11-15$.

[16] T. Tian, H. Iwai, and H. Sasaoka, "Pseudo BER based snr estimation for energy detection scheme in cognitive radio," in IEEE 75th Veh. Technol. Conf., May 2012, pp. 1 -5.

[17] H. Hui, "Influence of antenna characteristics on MIMO systems with compact monopole arrays," IEEE Antennas and Wireless Propag. Letters, vol. 8, pp. $133-136,2009$

[18] D.-S. Shiu, G. Foschini, M. Gans, and J. Kahn, "Fading correlation and its effect on the capacity of multielement antenna systems," IEEE Trans. Commun., vol. 48, no. 3, pp. 502 -513, Mar. 2000.

[19] S. Kim, J. Lee, H. Wang, and D. Hong, "Sensing performance of energy detector with correlated multiple antennas," IEEE Signal Process. Letters, vol. 16, no. 8, pp. 671 -674, Aug. 2009.

[20] V. Banjade, N. Rajatheva, and C. Tellambura, "Performance analysis of energy detection with multiple correlated antenna cognitive radio in nakagami-m fading," IEEE Commun. Letters,, vol. 16, no. 4, pp. 502 -505 , April 2012.

[21] S. K. Sharma, S. Chatzinotas, and B. Ottersten, "Eigenvalue based sensing and SNR estimation for cognitive radio in presence of noise correlation," IEEE Trans. Veh. Technol., vol. 62, no. 8, pp. 1-14, 2013.

[22] R. Janaswamy, "Effect of element mutual coupling on the capacity of fixed length linear arrays," IEEE Antennas and Wireless Propag. Letters, vol. 1 , no. 1 , pp. $157-160,2002$.

[23] D. Voiculescu, "Multiplication of certain non-commuting random variables," J. Operator Theory, vol. 18, pp. 223-235, 1987.

[24] - "Addition of certain non-commuting random variables," J. Funct. Analysis, vol. 66, pp. 323-346, 1986

[25] S. K. Sharma, S. Chatzinotas, and B. Ottersten, "SNR Estimation for Multi-dimensional Cognitive Receiver under Correlated Channel/Noise", IEEE Trans. Wireless Commun., 2013, submitted.

[26] Y.-C. Chen and Y.-T. Su, "MIMO channel estimation in correlated fading environments," IEEE Trans. Wireless Commun., vol. 9, no. 3, pp. 11081119, 2010. 